\title{
Evaluation of resin infiltration on demineralized root surface: An in vitro study
}

\author{
Yuan ZHOU ${ }^{1}$, Khairul MATIN ${ }^{1,2}$, Yasushi SHIMADA ${ }^{1}$, Yasunori SUMI ${ }^{3}$ and Junji TAGAMI ${ }^{1}$ \\ ${ }^{1}$ Cariology and Operative Dentistry, Department of Oral Health Sciences, Graduate School of Medical and Dental Sciences, Tokyo Medical and \\ Dental University, 1-5-45 Yushima, Bunkyo-ku, Tokyo 113-8549, Japan \\ ${ }^{2}$ Endowed Department of International Oral Health Science (affiliated with Department of Translational Research), School of Dental Medicine, \\ Tsurumi University, 2-1-3 Tsurumi, Tsurumi-ku, Yokohama 230-8501, Japan \\ ${ }^{3}$ Division of Oral and Dental Surgery, Department of Advanced Medicine, National Hospital for Geriatric Medicine, National Center for Geriatrics and \\ Gerontology, 36-3, Gengo, Morioka, Obu, Aichi 474-8511, Japan \\ Corresponding author, Yasushi SHIMADA; E-mail: shimada.ope@tmd.ac.jp
}

\begin{abstract}
The aim of this study was to evaluate the effect of resin infiltration on root caries induced by Streptococcus mutans biofilms. Human premolar specimens were divided to 5 groups: negative control (NC), Clearfil SE Bond (SEB), Icon-etch120s+Icon-infiltrant (HA120), Icon-etch10s+Icon-infiltrant (HA10) and K-etchant10s+Icon-infiltrant (PA10). The resin penetration was observed by fluorescent microscope. Biofilm-induced demineralization was conducted again and observed by swept-source optical coherence tomography and confocal laser scanning microscope. The maximum resin penetration depth (PDmax), lesion depth increase ( $\triangle \mathrm{LD}$ ), frequency of cervical enamel loss and dentinoenamel junction separation length were measured and statistically analyzed. HA120 showed $138.00 \pm 49.25 \mu \mathrm{m}$ PDmax that was significantly larger than PA10 and SEB $(p<0.05)$. SEB created $136.58 \pm 64.73 \mu \mathrm{m}$ coating layers. HA120 and SEB showed significantly lower $\triangle \mathrm{LD}$ than NC $(p<0.05)$. Resin infiltration with $120 \mathrm{~s}-\mathrm{HCl}$ pretreatment has got a good penetration ability and preventive effect on root caries, however, an additional risk factor of cervical enamel loss was identified.
\end{abstract}

Keywords: Resin infiltration, Root caries, Hydrochloric acid, Phosphoric acid, Biofilm

\section{INTRODUCTION}

Root caries usually occurs where root dentine is exposed to oral environment as a result of gingival recession, which may be caused by mechanical toothbrush trauma or chronic periodontitis ${ }^{1)}$. Root caries increases in prevalence with age and will precipitate the breakdown of remaining natural and restored teeth ${ }^{2)}$. Because cementum has half as much mineral as enamel by weight, demineralization on the root surface is approximately twice as rapid as that on enamel and can occur at a higher $\mathrm{pH}^{3)}$. Additionally, root dentine surfaces may even present a high risk to caries development ${ }^{4)}$ due to a low salivary flow, which is frequently found in elderly as a side-effect of daily use of medicines ${ }^{5}$. Various treatment modalities have been proposed for the management of root caries, including the improvement of oral conditions for oral hygiene, topical application of remineralization agents and antimicrobial regimen ${ }^{6}$, and coating or sealing by resin-based materials ${ }^{7}$. However, efficient and simple methods, which can protect the exposed root surface from carious attack for a long term and for elderly patients requiring home care, are not yet currently available ${ }^{8}$.

In recent years, a micro-invasive treatment with a low-viscosity resin-infiltrant has become one of the options for non-cavitated enamel caries. Resin infiltration on enamel caries has been clinically proven to effectively arrest and stabilize lesions ${ }^{9-11)}$. It has also been proven to significantly increase micro-hardness and

Color figures can be viewed in the online issue, which is available at J-STAGE.

Received Jun 29, 2016: Accepted Oct 11, 2016

doi:10.4012/dmj.2016-229 JOI JST.JSTAGE/dmj/2016-229 reduce mineral loss after a demineralization challenge compared with untreated lesions ${ }^{12}$. The mechanism is that resin-infiltrant exploits capillary forces to transport resins with high penetration coefficients into enamel porosities, and after polymerization the infiltrant occludes diffusion pathways for cariogenic acids and dissolved minerals ${ }^{13)}$. Resin-infiltrant has a significantly deeper penetration in the lesion body than conventional dental adhesives ${ }^{14,15)}$ and the diffusion barrier of caries infiltration is not formed at the lesion surface as caries sealing and coating but created inside the lesion body ${ }^{16)}$. Besides that, caries infiltrants are optimized for rapid capillary penetration and exhibit a very low-viscosity, low contact angle to enamel and high surface tension ${ }^{16)}$. While resin infiltration was efficacious in preventing further demineralization of artificial enamel caries lesions under cariogenic conditions in situ ${ }^{17)}$, its potential effect on root caries has not been studied so far.

A technique called optical coherence tomography (OCT) has been developed for noninvasive crosssectional imaging of internal biological structures ${ }^{18)}$. In dentistry, OCT was introduced for the evaluation of tooth demineralization and remineralization ${ }^{19,20)}$. A recent study also showed that OCT could quantitatively evaluate the penetration of resin-infiltrant into early dental caries ${ }^{21)}$.

The aim of this study was to evaluate the potential effect of resin infiltration with different pretreatment conditions on root caries induced by cariogenic bacterial biofilms in vitro. The null hypothesis was that resin infiltration could not reduce further demineralization of root caries lesions under cariogenic conditions. 


\section{MATERIALS AND METHODS}

\section{Specimen preparation}

Thirty extracted intact human premolars with no visible evidence of caries or cracks were collected after obtaining the informed consent according to a protocol approved by the Institutional Review Board of Tokyo Medical and Dental University (approval no. 725). The teeth were stored in $4^{\circ} \mathrm{C}$ in water containing $0.1 \%$ wt of thymol in order to inhibit bacterial growth for a maximum of 6 months until use. The upper two-third crown and the lower two-third root of the teeth were cut by a lowspeed diamond saw (Isomet, Buehler, Lake Bluff, IL, USA), keeping $2 \mathrm{~mm}$ crown and $3 \mathrm{~mm}$ root away from the enamel-cementum junction (Fig. 1). Then the buccal and lingual cervical parts were vertically cut into half. Sixty square-shaped blocks $(5 \times 4 \times 2 \mathrm{~mm})$ were made by a diamond bur (FG 102R, Shofu, Kyoto, Japan) attached to an air turbine headpiece under copious cooling water. Two semi-round holes (depth: $0.5 \mathrm{~mm}$ ) were marked by a diamond bur (D4010f, GC, Tokyo, Japan) on the upper and lower central top surface of each specimen. The specimens were observed by swept-source optical coherence tomography (SS-OCT, IVS-2000, Santec, Komaki, Japan). Then five cutting surfaces were covered with a thin layer of acid-resistant varnish (Shiseido, Tokyo, Japan) and one edge of the top surface was covered by 1-mm-wide paraffin wax (GC) to keep the baseline from being demineralized.

In vitro root caries formation (First time demineralization) In vitro biofilms were formed on the tooth surfaces using a laboratory strain of oral cariogenic bacteria Streptococcus mutans (S. mutans) MT8148. A suspension of $S$. mutans at an optical density of 2.5 at $500 \mathrm{~nm}\left(\mathrm{OD}_{500}=2.5\right)$ was prepared from a 16-h fresh culture in Brain Heart Infusion (BHI, Becton Dickinson, Sparks, MD, USA) broth. The suspension was washed three times with phosphate buffered saline (PBS) and stored at $4^{\circ} \mathrm{C}$ with gentle stirring. For growing biofilms, a solution of Heart Infusion (HI, Becton Dickinson, Sparks, MD, USA) broth with sucrose (at 1-2\% final concentration) was used.

The biofilms were formed in an oral biofilm reactor (OBR) according to the previously reported method ${ }^{22,23)}$. In brief, OBR is equipped with two chambers, with each chamber containing a warm water jacket to maintain a constant interior temperature, to grow biofilms under anaerobic conditions for $24 \mathrm{~h}$ (Fig. 2). A flat-bulb $\mathrm{pH}$ electrode is used to monitor the $\mathrm{pH}$ beneath the biofilm continuously. The specimens with undisturbed biofilms were further inoculated for three days to produce root caries lesions. All the specimens were kept in separate wells of a 24-well culture plate (Corning, NY, USA) at $37^{\circ} \mathrm{C}$ and sucrose-HI was supplemented every day.

After a 3-day incubation, specimens were cleaned using $0.25 \mathrm{M} \mathrm{NaOH}$ and washed with distilled water to remove biofilms. The paraffin wax was removed gently. Then the specimens were observed by SS-OCT.

\section{Materials application}

The composition of materials used in this study was shown in Table 1 . The specimens were randomly divided

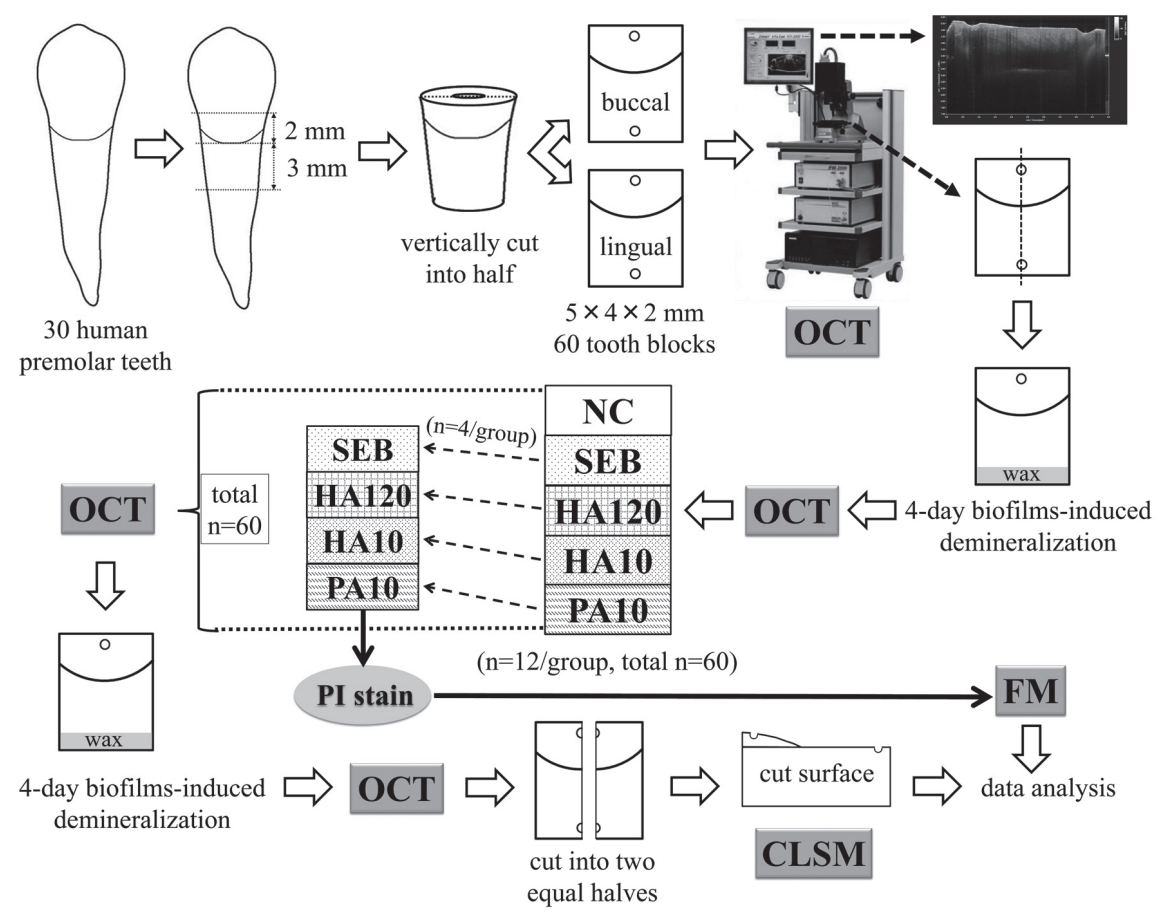

Fig. 1 Schematic diagram of the study design and methodology. 


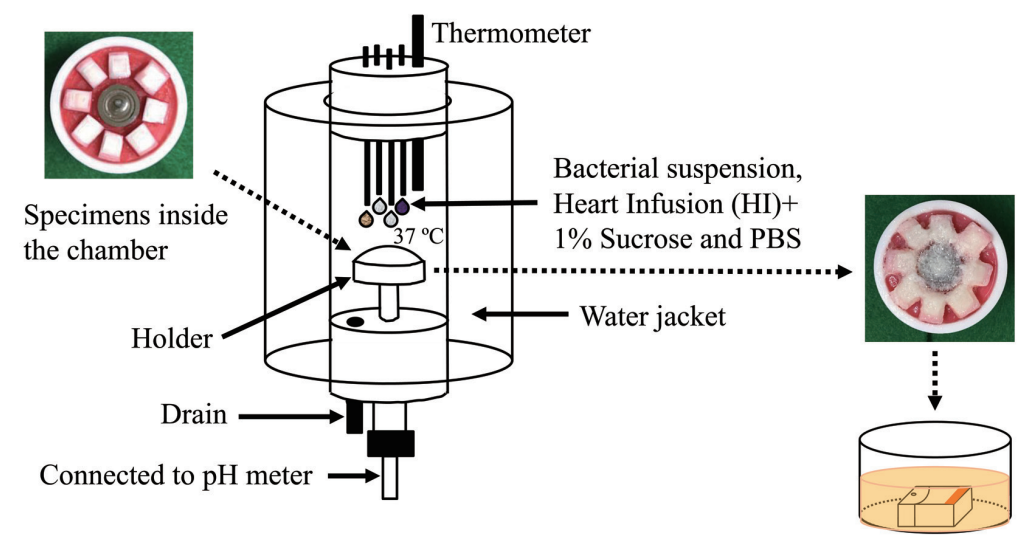

Oral biofilm reactor (OBR) for 24 hours (for primary adhesion and growth of biofilm)

Incubation for 3 days at $37^{\circ} \mathrm{C}$

Fig. 2 Diagram of one of the chambers of the oral biofilm reactor (OBR) within which artificial biofilms were formed on the specimens surfaces.

Digital photographs were the specimens before (left) and $24 \mathrm{~h}$ after biofilm formation (right).

Table 1 Materials used in this study

\begin{tabular}{|c|c|c|c|c|}
\hline Material & Code & Composition & Lot No. & Manufacturer \\
\hline Clearfil SE Bond & SEB & $\begin{array}{l}\text { Primer: } \\
\text { MDP, HEMA, hydrophilic } \\
\text { aliphatic dimethacrylate, dl-CQ, } \\
\text { N,N-Diethanol-p-toluidine, water } \\
\text { Bond: } \\
\text { MDP, Bis-GMA, HEMA, hydrophobic } \\
\text { aliphatic dimethacrylate, dl-CQ, } \\
\text { N,N-Diethanol-p-toluidine, colloidal silica }\end{array}$ & $8 \mathrm{~K} 0047$ & $\begin{array}{l}\text { Kuraray Noritake Dental, } \\
\text { Tokyo, Japan }\end{array}$ \\
\hline Icon-Etch & HA & $\begin{array}{l}15 \% \mathrm{HCl} \text {, pyrogenic silicic acid, } \\
\text { surface-active agents }\end{array}$ & 692332 & DMG, Hamburg, Germany \\
\hline Icon-Dry & & $99 \%$ ethanol & 692328 & DMG \\
\hline Icon-Infiltrant & & $\begin{array}{l}\text { methacrylate-based resin matrix } \\
\text { (TEGDMA), initiators, additives }\end{array}$ & 692325 & DMG \\
\hline K-etchant gel & PA & $40 \% \mathrm{H}_{3} \mathrm{PO}_{4}$, water, colloidal silica, dye & 470022 & Kuraray Noritake Dental \\
\hline Propidium iodide & PI & $\begin{array}{l}\text { red component used for inftration } \\
\text { test of LIVE/DEAD BacLight } \\
\text { Bacterial Viability Kit }\end{array}$ & 1702 & $\begin{array}{l}\text { Molecular Probes, Invitrogen } \\
\text { Detection Technologies, } \\
\text { Carlsbad, CA, USA }\end{array}$ \\
\hline
\end{tabular}

MDP: 10-methacryloyloxydecyl dihydrogen phosphate; HEMA: 2-hydroxyethyl methacrylate; CQ: camphorquinone; BisGMA: bisphenol-A-diglycidyl methacrylate; TEGDMA: triethyleneglycol dimethacrylate

into 5 groups with 12 specimens in each and were treated as follows:

(1) NC: served as a negative control group without using any materials.

(2) SEB: treated by Clearfil SE Bond (Kuraray Noritake Dental, Tokyo, Japan). The surface was applied by the primer for $20 \mathrm{~s}$ and mild air was blown. The bond was applied and air was gently blown. Then they were irradiated with a halogen light-curing unit with $600 \mathrm{~mW} / \mathrm{cm}^{2}$ output (Optilux 501, Kerr, Orange, CA, USA) for $10 \mathrm{~s}$.

(3) HA120: treated by 120 s $15 \% \mathrm{HCl}$ (Icon-etch, DMG, Hamburg, Germany), 99\% ethanol (Icon- 
dry, DMG) and a low-viscosity resin (Iconinfiltrant, DMG). The surface was etched with Icon-etch for $120 \mathrm{~s}$, rinsed for $30 \mathrm{~s}$ and dehydrated with Icon-dry for $30 \mathrm{~s}$. Icon-infiltrant was applied on the specimens with a sponge. After $3 \mathrm{~min}$, gentle air was blown. The resin was light-cured (Optilux 501, Kerr) for $40 \mathrm{~s}$ at $600 \mathrm{~mW} / \mathrm{cm}^{2}$. Iconinfiltrant was then applied a second time for an additional $1 \mathrm{~min}$ and light-cured for $40 \mathrm{~s}$.

(4) HA10: treated by $10 \mathrm{~s} 15 \% \mathrm{HCl}$ (Icon-etch), $99 \%$ ethanol (Icon-dry) and a low-viscosity resin (Iconinfiltrant). Except the etching time, other steps were the same as the application of group (3).

(5) PA10: treated by $10 \mathrm{~s} 40 \% \mathrm{H}_{3} \mathrm{PO}_{4}$ (K-etchant gel, Kuraray Noritake Dental), 99\% ethanol (Icondry) and a low-viscosity resin (Icon-infiltrant). Except the etching material and etching time, other steps were the same as the application of group (3).

\section{Fluorescent microscope (FM) observation}

In order to observe the resin thickness and the resin penetration depth, 4 specimens in group (2) were applied by the mixture of the bond of SE Bond and propidium iodide (PI, LIVE/DEAD BacLight Bacterial Viability Kit, Molecular Probes, Invitrogen Detection Technologies, Carlsbad, CA, USA), and 4 specimens in group (3) to (5) were applied by the mixture of Icon-infiltrant and PI as pretreatments. The left $0.5 \mathrm{~mm}$ cutting surface of the stained specimens were gently trimmed off using wet 2000-grit silicon carbide papers to expose a fresh cross section. Then they were observed by fluorescent microscope (FM). A custom code in the image analysis software (Image J, version 1.48, National Institutes of Health, Bethesda, MD, USA) was used to measure the maximum resin thickness and the maximum resin penetration depth (PDmax) (Fig. 3). Then the specimens were observed by SS-OCT.

\section{Demineralization challenge of materials (Second time} demineralization)

One edge of the top surface of root dentin was covered again by 1 -mm-wide paraffin wax to keep the baseline from being demineralized. All specimens were demineralized again the same way as mentioned above. After a three-day incubation, specimens were cleaned using $0.25 \mathrm{M} \mathrm{NaOH}$ and washed with distilled water to remove biofilms. The paraffin wax was removed gently. Then the specimens were observed by SS-OCT.

\section{Swept-source optical coherence tomography (SS-OCT) observation}

SS-OCT (IVS-2000, Santec) was used to examine every step of the experiment process. The stages were labeled as DeM0 (before demineralization), DeM1 (after the 1 st time demineralization), Mat (after immediate materials application) and DeM2 (after the 2nd time demineralization). This system is a frequency-domain OCT technique that interprets the magnitude and coherence of the light reflected from the subject into the depth-profile of the subject. The system incorporates a high-speed frequency, swept external-cavity laser. The wavelength ranged from 1,260 to $1,360 \mathrm{~nm}$ (centered at $1,310 \mathrm{~nm}$ ) at a $20-\mathrm{kHz}$ sweep rate. The optical resolution was $20 \mu \mathrm{m}$ transversally and $12 \mu \mathrm{m}$ axially in the air (7-8 $\mu \mathrm{m}$ in tissues with a refractive index around 1.5). Every time, SS-OCT examination was performed along the same plane between the marked two holes and then central cross-section images were obtained. The demineralized specimens were cross-sectionally scanned with SS-OCT after gentle blot-drying of the surface leaving it moist without any visible water-droplets. To ensure the reproducibility of the scan, the specimens were placed at the same orientation as accurately as

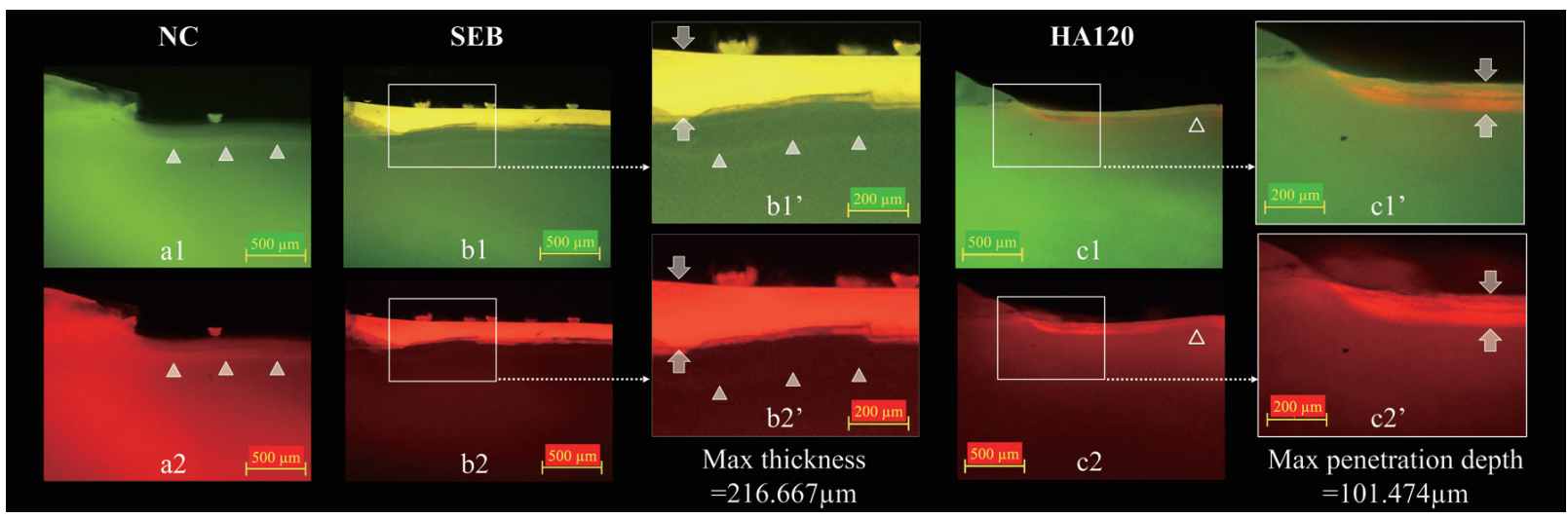

Fig. 3 FM images of the lesions without any treatment in NC (a1, a2), treated by SE Bond (b1, b2) and treated by resin-infiltrant with $120 \mathrm{~s} \mathrm{HCl}$ pretreatment (c1, c2).

The solid triangles in a1, a2, b1' and b2' showed the root dentin lesion layers. The arrows in b1' and b2' showed the maximum resin thickness of SEB $(216.667 \mu \mathrm{m})$. The blank triangles in $\mathrm{c} 1$ and $\mathrm{c} 2$ showed the incomplete stained resin penetration areas. The arrows in c1' and c2' showed the maximum resin penetration depth in HA120 (101.474 $\mu \mathrm{m})$. 
possible.

A custom code in the image analysis software (Image J) was used to read the raw data of SS-OCT. The obtained SS-OCT image was rotated to compensate for the tilting during the scan to reach a horizontal surface. A binarization process (Fig. 4) was applied ${ }^{24)}$. The linear measurement of lesion depth after the 1st time demineralization ( $\mathrm{LD} 1_{\mathrm{OCT}}$ ) and the 2nd time demineralization (LD2 ${ }_{\mathrm{OCT}}$ ) were shown in Fig. 4. The increase in lesion depth $(\Delta \mathrm{LD})$ was calculated as:
$\Delta \mathrm{LD}=\mathrm{LD} 2_{\mathrm{OCT}}-\mathrm{LD} 1_{\mathrm{OCT}}$. The length of dentino-enamel junction (DEJ) separation was measured and the frequency of specimens with cervical enamel loss was counted.

Confocal laser scanning microscope (CLSM) observation After the SS-OCT observation of the 2nd demineralization stage, all specimens were fixed in epoxy resin (EpoxiCure, Buehler). After 8 h, a low-speed diamond saw (Isomet) was used to cut each specimen in

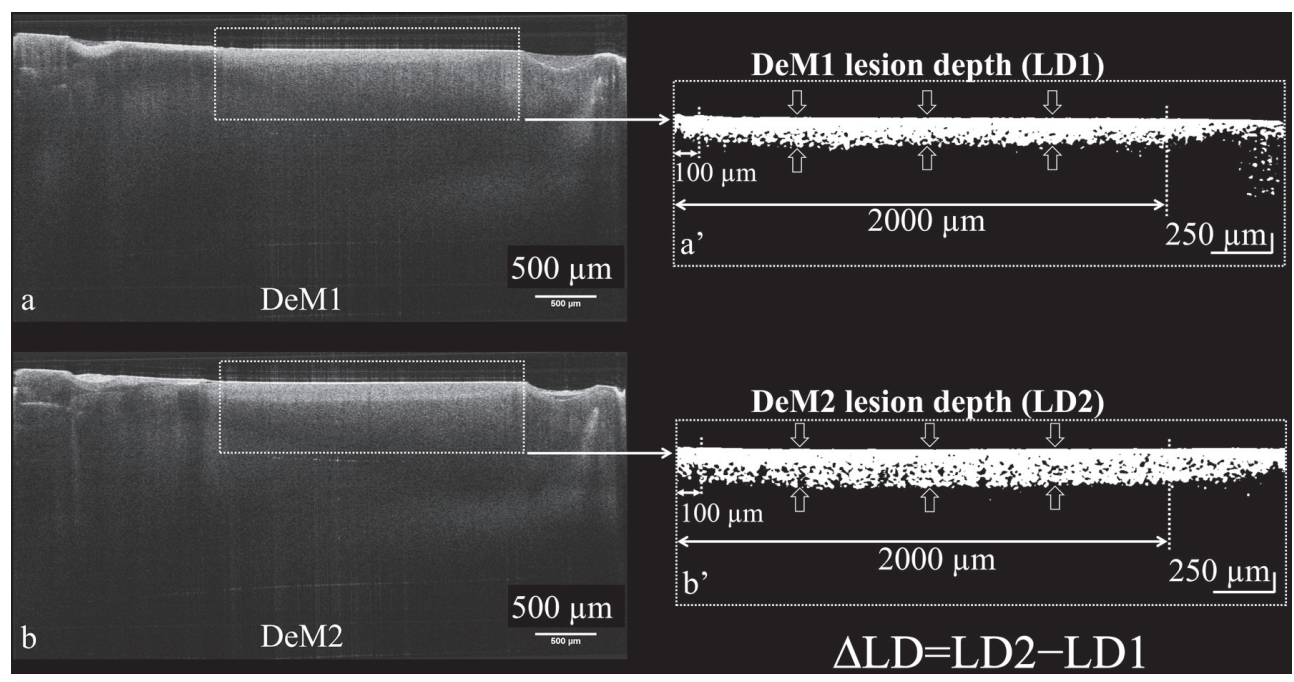

Fig. 4 Linear measurement of lesion depth from SS-OCT images.

(a) Image after the 1st demineralization (DeM1). (b) Image after the 2nd demineralization (DeM2). The obtained images were rotated to compensate for the tilting during the scan to reach a horizontal surface. Cervical areas of interest were selected. A noise-reducing median filter (size 2) and a binarization process were applied to the raw data (a', b'). The blank arrows showed the lesion layers. The lesion depth was measured vertically from the surface to the bottom of the lesion. The lesion depths of 20 positions from each specimen were measured with a width of $2,000 \mu \mathrm{m}$ and an interval of $100 \mu \mathrm{m}$. The average was calculated as the value of lesion depth of one specimen. LD1 was the lesion depth after DeM1. LD2 was the lesion depth after DeM2. $\triangle \mathrm{LD}$ was the increase in lesion depth from LD1 to LD2.

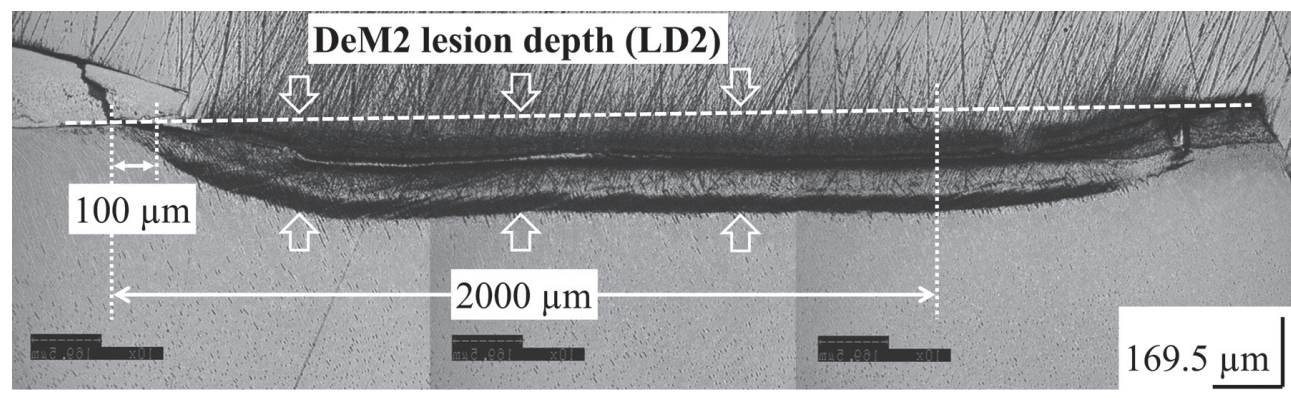

Fig. 5 Linear measurement of lesion depth from CLSM images.

A baseline was drawn from the dentino-enamel junction to the intact root surface which was covered by paraffin wax during demineralization. The lesion depth was measured vertically from the surface to the bottom of the lesion. The lesion depths of 20 positions from each specimen were measured with a width of $2,000 \mu \mathrm{m}$ and an interval of 100 $\mu \mathrm{m}$. The average was calculated as the value of lesion depth of one specimen. 
half along the two central holes and obtain discs with thickness of approximately $2 \mathrm{~mm}$. Then, the slices were trimmed off using wet 2000-grit silicon carbide papers and further polished with diamond paste down to 0.25 $\mu \mathrm{m}$ under running water. The cross-sectional slice examined using the nondestructive SS-OCT imaging was physically separated and examined under CLSM (1LM21H/W, Lasertec, Yokohama, Japan). The lesion

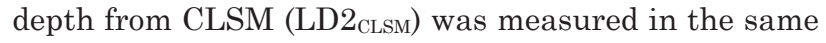
manner as that from SS-OCT by Image J (Fig. 5).

\section{Dentin surface removal by pretreatments}

Additional specimens were prepared for observing the amount of normal dentin surface removal by different pretreatments. Four intact human root surfaces were polished by wet 2000-grit silicon carbide papers to expose flat and fresh root dentin surfaces $(4 \times 4 \times 3 \mathrm{~mm})$. After cleaning by tap water, half surfaces were covered by stick tapes to keep the baselines and another half surfaces were applied by $15 \% \mathrm{HCl}$ (Icon-Etch) $120 \mathrm{~s}, 15 \%$ $\mathrm{HCl}$ (Icon-Etch) $10 \mathrm{~s}, 40 \% \mathrm{H}_{3} \mathrm{PO}_{4}$ (K-etchant gel) $10 \mathrm{~s}$ and SE Bond primer $20 \mathrm{~s}$, respectively. Then the tapes were removed and air was blown according to manufactory instructions. After SS-OCT observation, the specimens were fixed in epoxy resin and cut in half by a low-speed diamond saw. The cross-sectioned surfaces were polished and observed by CLSM. The dentin surface removal was measured vertically from the baseline to the eroded surface by Image J.

\section{Statistical analysis}

The maximum resin penetration depth (PDmax) and the increase in lesion depth $(\Delta \mathrm{LD})$ were analyzed by one-way ANOVA with Tukey's post hoc tests. The correlation between $\mathrm{LD} 2_{\mathrm{OCT}}$ and $\mathrm{LD} 2_{\mathrm{CLSM}}$ was analyzed by Pearson's correlation test. The DEJ

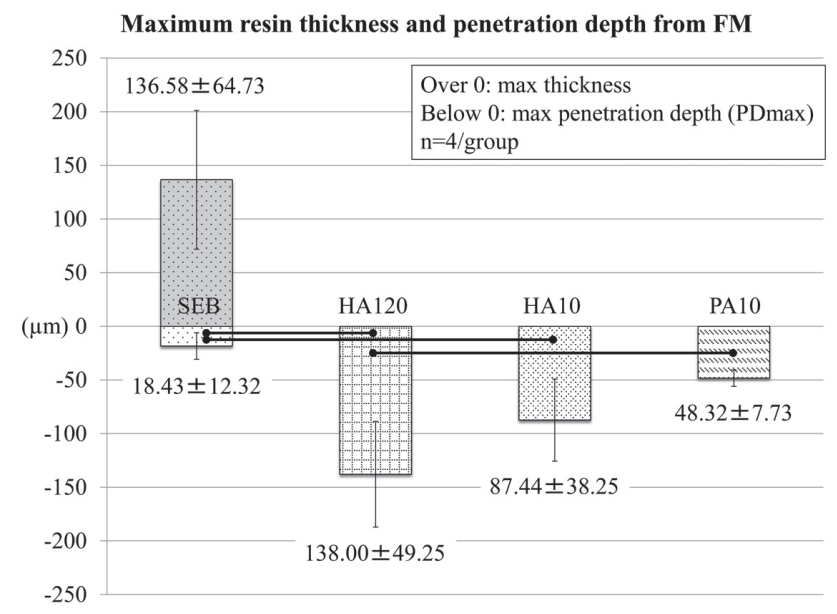

Fig. 6 Maximum resin thickness and maximum resin penetration depth (PDmax) from FM analysis.

Bars showed significant differences among groups in PDmax $(p<0.05$, one-way ANOVA, Tukey's post hoc test). separation length of DeM and that of Mat was compared by Wilcoxon signed ranks test. The frequency of specimens with cervical enamel loss of DeM1 and that of Mat was analyzed by Fisher's exact test. All statistical analyses were performed with $95 \%$ level of confidence using Statistical Package for Medical Science (Version 16.0 for Windows, SPSS, Chicago, IL, USA).

\section{RESULTS}

The penetrated resin in the lesion body by the treatment of Icon-infiltrant and SE Bond could be detected from FM (Fig. 3). HA120 showed 138.00 \pm 49.25 $\mu \mathrm{m}$ PDmax that was significantly larger than PA10 and SEB $(p<0.05)$. A maximum thickness of $136.58 \pm 64.73$ $\mu \mathrm{m}$ coating layer in SEB could be detected on the lesion surface, while resin-infiltrant could not be detected on the lesion surface. Moreover, resin-infiltrant formed inhomogeneous penetration layers in demineralized dentin, while SE Bond formed homogeneous coating layers, which were stable even after the 2nd demineralization (Figs. 3 and 6).

The SS-OCT images were taken in wet condition, and the lesion surface appeared flat with no remarkable shrinkage or loss of surface as presented in Fig. 4. In all the SS-OCT images after demineralization, a boundary was seen below the lesion surface that suggested the lesion front as bright zones with increased signal intensity. LD2 $2_{\mathrm{OCT}}$ was remarkably higher than LD1 $1_{\mathrm{OCT}}$ in all groups (Figs. 4 and 7). HA120 and SEB showed significantly lower $\triangle \mathrm{LD}$ than $\mathrm{NC}(p<0.05)$, while HA10 and PA10 did not show significantly lower $\Delta \mathrm{LD}$ than NC $(p>0.05)$ (Fig. 7). LD2 $2_{\text {OCT }}$ and LD2 $2_{\text {CLSM }}$ showed a significant correlation $(\mathrm{r}=0.865, p<0.05)$ (Fig. 8).

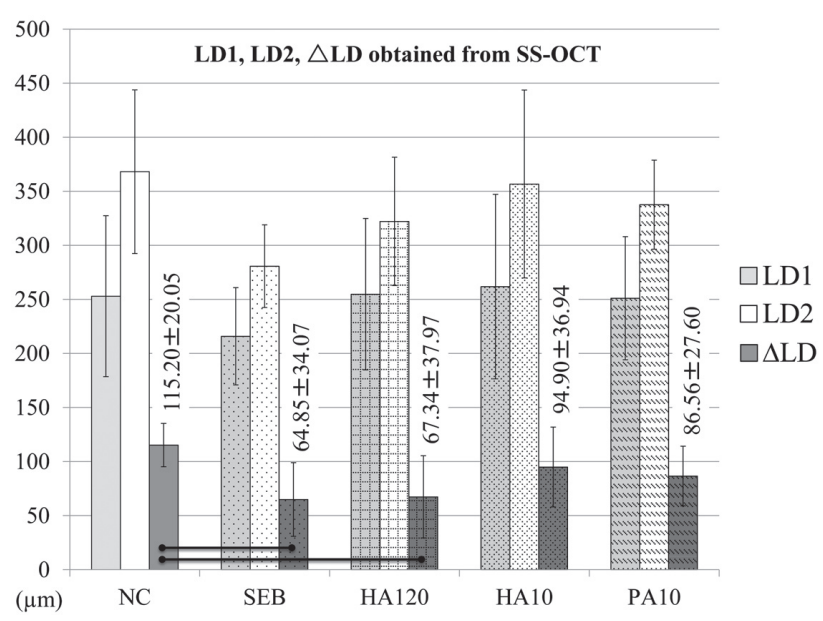

Fig. 7 The lesion depth after the 1st time demineralization (LD1) and that after the 2nd time demineralization (LD2) and the increase in lesion depth $(\Delta \mathrm{LD})$ from SS-OCT analysis.

Bars showed significant differences among groups in $\Delta \mathrm{LD}(p<0.05$, one-way ANOVA, Tukey's post hoc test). 
DEJ separation showed on SS-OCT images as a white line with intensified brightness and changed in length during different experimental stages. The length

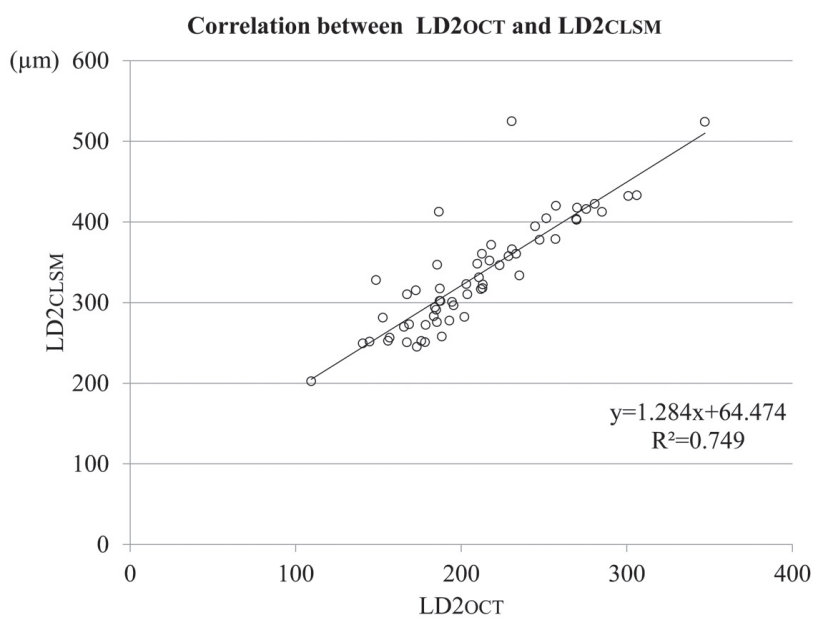

Fig. 8 Significant correlation between LD2 ${ }_{\text {OCT }}$ and LD2 $2_{\text {CLSM }}$ $(\mathrm{r}=0.865, p<0.05$, Pearson's correlation test).

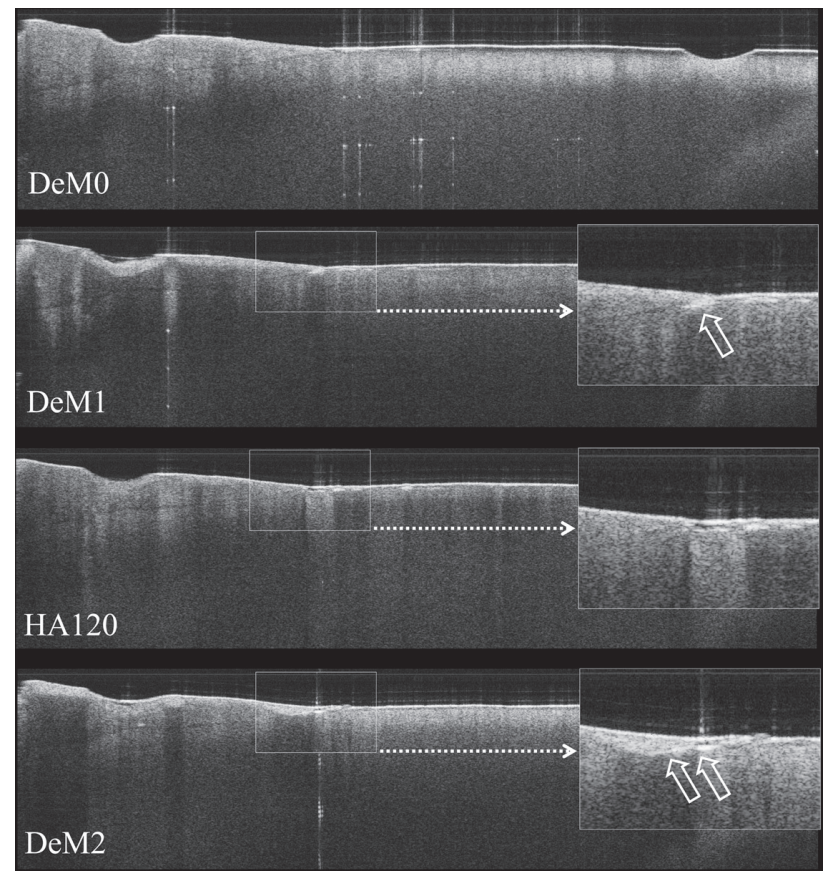

Fig. 9 DEJ separation changed in length during different experimental stages.

The blank arrows showed the DEJ separation. After the 1st demineralization (DeM1), DEJ separation formed and showed on SS-OCT images as a white line with increased signal intensity. After immediate application of resin-infiltrant with $120 \mathrm{~s} \mathrm{HCl}$ pretreatment (HA120), DEJ separation disappeared. However, after the 2nd demineralization (DeM2), DEJ separation formed again and increased in length. of DEJ separation reduced in HA120, HA10 and PA10, while that increased in SEB after immediate materials application. In HA120, DEJ separation length of Mat

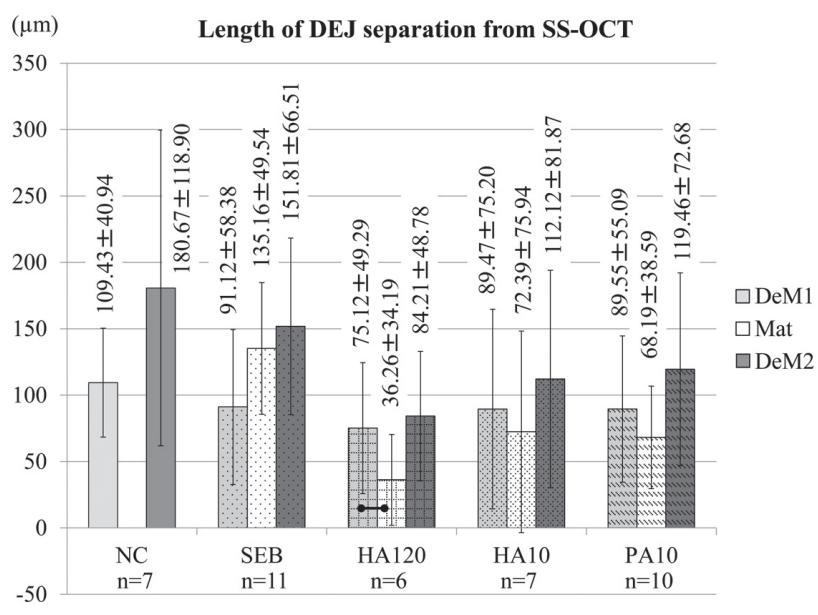

Fig. 10 Length of DEJ separation from SS-OCT analysis. The length of DEJ separation was compared between DeM1 and Mat within each group of SEB, HA120, HA10 and PA10. Bars showed significant differences between DeM1 and Mat within each group ( $p<0.05$, Wilcoxon signed ranks test). DeM1: after the 1st time demineralization; Mat: after immediate materials application; DeM2: after the 2nd time demineralization.

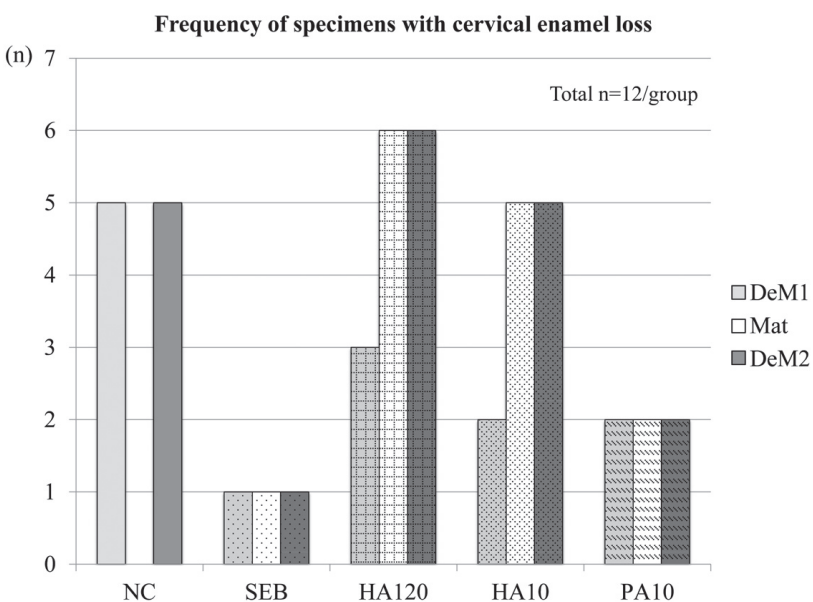

Fig. 11 The frequency of specimens with cervical enamel loss from SS-OCT analysis.

The frequency was compared between DeM1 and Mat within each group of SEB, HA120, HA10 and PA10. No significant difference was detected between DeM1 and Mat within each group ( $p>0.05$, Fisher's exact test). DeM1: after the 1st time demineralization; Mat: after immediate materials application; DeM2: after the 2nd time demineralization. 


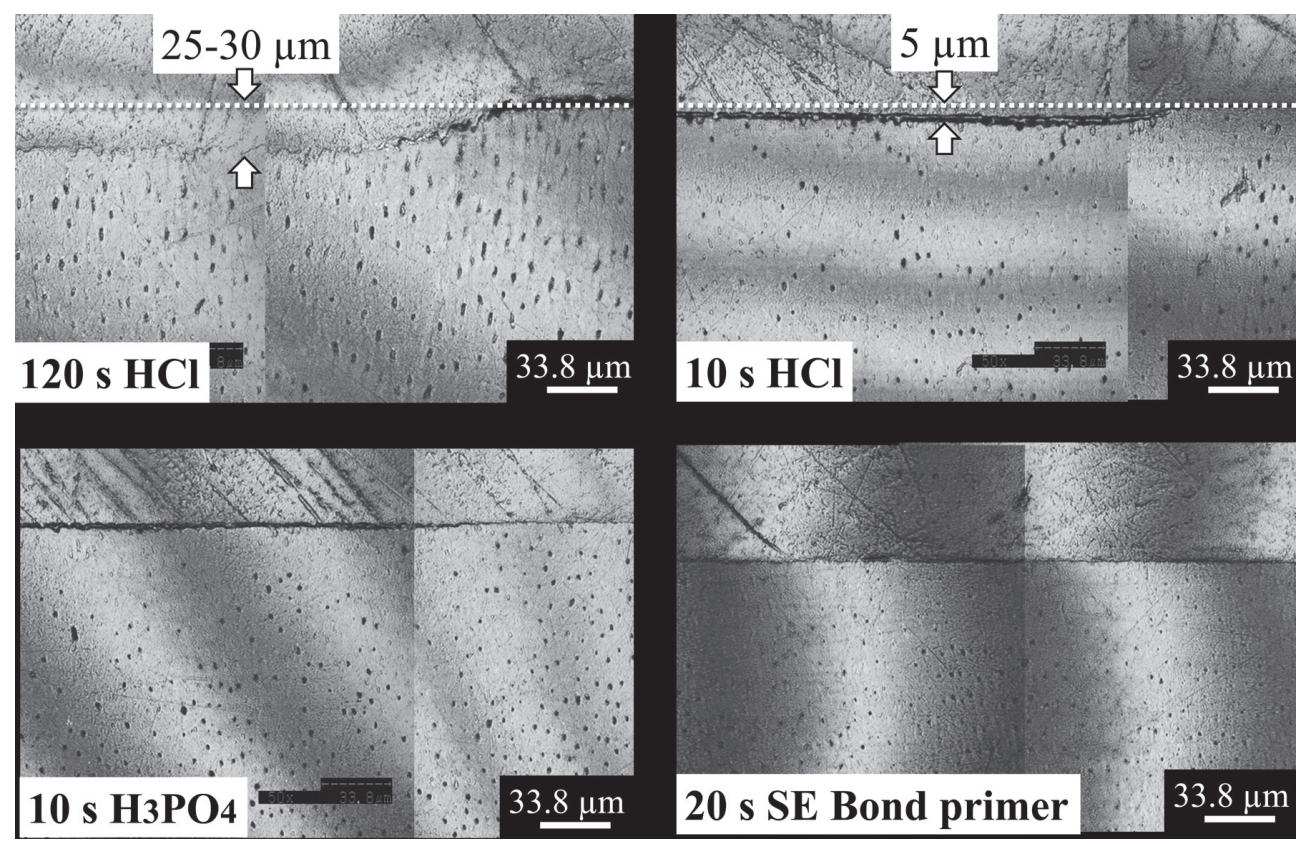

Fig. 12 CLSM images of surface removal of normal human root dentin with different pretreatments.

The dot line was the baseline. After etching with $15 \% \mathrm{HCl}$ (Icon-etch) for $120 \mathrm{~s}$, approximately $25-30 \mu \mathrm{m}$ dentin surface was removed. After etching with $15 \% \mathrm{HCl}$ (Icon-etch) for $10 \mathrm{~s}$, approximately $5 \mu \mathrm{m}$ dentin surface was removed. After etching with $40 \% \mathrm{H}_{3} \mathrm{PO}_{4}$ (K-etchant) for $10 \mathrm{~s}$, dentin surface removal could not be detected. After applying by SE Bond primer for $20 \mathrm{~s}$, dentin surface removal could not be detected.

was significantly lower than that of DeM1 $(p<0.05)$. However, in groups SEB, HA10 and PA10, DEJ separation length did not show significant difference between DeM1 and Mat within each group $(p>0.05)$ (Figs. 9 and 10).

From SS-OCT images, although no significate difference in the frequency of specimens with cervical enamel loss was detected between DeM1 and Mat within each group $(p>0.05)$, the frequency increased in HA120 and HA10 after immediate materials application. Finally, approximately half specimens of HA120 and HA10 and only one specimen of SEB had cervical enamel loss (Fig. 11).

The thicknesses of normal root dentin surface removal by different pretreatments were ordered by follows: $15 \% \mathrm{HCl} 120 \mathrm{~s}(25-30 \mu \mathrm{m})>15 \% \mathrm{HCl} 10 \mathrm{~s}(5$ $\mu \mathrm{m})>40 \% \mathrm{H}_{3} \mathrm{PO}_{4} 10 \mathrm{~s}(0 \mu \mathrm{m})=\mathrm{SE}$ Bond primer $10 \mathrm{~s}(0$ $\mu \mathrm{m})$ (Fig. 12).

\section{DISCUSSION}

Resin infiltration has been introduced as an alternative treatment option for non-cavitated enamel lesions that are not expected to remineralize or arrest by non-invasive measures alone ${ }^{25)}$. It could effectively prevent the further demineralization of artificial enamel caries lesions under cariogenic conditions in $s i t u^{17)}$. On the other hand, with the increase of a dentate elderly population, the occurrence of root caries is also increasing. Preventing and arresting root caries is one of the essential issues to maintain the quality of life of elderly people ${ }^{26)}$. Therefore, the potential effect of resin infiltration on root caries was evaluated in this in vitro study. As a comparison, a conventional resin adhesive without composite resin filling was used to seal or to coat on root caries.

In this study, the null hypothesis that resin infiltration could not reduce further demineralization of root caries lesions under cariogenic conditions was rejected, because resin infiltration pretreated by $120 \mathrm{~s}$ $\mathrm{HCl}$ was shown to have a significantly less increase in lesion depth than the control group according to SS-OCT analysis. Whereas, resin infiltration pretreated by $10 \mathrm{~s}$ $\mathrm{HCl}$ and $10 \mathrm{~s} \mathrm{H}_{3} \mathrm{PO}_{4}$ showed similar lesion depth increase as the control group. Also, resin-infiltrant with $\mathrm{HCl}$ pretreatments could penetrate deeper than that with $\mathrm{H}_{3} \mathrm{PO}_{4}$ pretreatment and $\mathrm{SE}$ Bond. Resin infiltration results in considerably deeper resin penetration whilst the pre-treatment with hydrochloric acid seems to be more suitable compared with the use of a phosphoric $\operatorname{acid}^{27)}$. However, the application of $15 \% \mathrm{HCl}$ for $120 \mathrm{~s}$ apparently removed more dentin structure from the root surface (approximately 25-30 $\mu \mathrm{m}$ ) than other pretreatments. Similar result was reported previously that $120 \mathrm{~s} 15 \%$ hydrochloric acid gel treatment could erode the surface layer more effectively (around 50 
$\mu \mathrm{m})$ than $37 \%$ phosphoric acid ${ }^{28}$. Therefore, our results implied that effective erosion of dentin lesion surface is necessary for resin-infiltrant to penetrate into the lesion body, and the deeper penetration of resin-infiltrant the less lesion progression in root caries.

It should be mentioned that in order to avoid damaging the specimens, the thickness of demineralized root surface removal or additional demineralization depth by different etching pretreatments was not measured. LD $1_{\text {ост }}$ was the outcome of the 1 st (single) biofilm-induced demineralization, whereas $\mathrm{LD} 2_{\mathrm{OCT}}$ was the final outcome of twice (double) biofilm-induced demineralization and different materials application with different pretreatments. Therefore, LD2 ост might increase considering additional demineralization caused by $\mathrm{HCl}$ etching. However, $\mathrm{LD} 2_{\text {OCт }}$ might reduce considering the demineralized dentin surface partially removed by $\mathrm{HCl}$. The results showed that $\mathrm{LD} 2_{\mathrm{OCT}}$ was remarkably higher than $\mathrm{LD} 1_{\text {OCT }}$ in all groups.

Resin infiltration with $120 \mathrm{~s} \mathrm{HCl}$ pretreatment showed a similar lesion depth increase as SE Bond. The former formed $138.00 \pm 49.25 \mu \mathrm{m}$ maximum resin penetration into the lesion body but did not form coating layers on the lesion surface. In reverse, the later formed $136.58 \pm 64.73 \mu \mathrm{m}$ maximum coating layers on the lesion surface, but only $18.00 \pm 12.36 \mu \mathrm{m}$ maximum resin penetration into the lesion body. This result was in accordance with previous studies showing that resininfiltrant has a significantly deeper penetration in the lesion body than conventional dental adhesives ${ }^{14,15}$. Finally, both of resin-infiltrant and SE Bond, based on different mechanisms, could reduce the further demineralization of root caries lesions in this in vitro study.

By observing and analyzing SS-OCT images of different experimental stages, we could find a tendency that DEJ separation reduced in length in three resininfiltrant groups, while they increased in length in SE Bond group from the 1st demineralization to the immediate materials application stage. DEJ separation, similar as an enamel crack, showed on SS-OCT images as a white line with intensified brightness or increased backscattering of light ${ }^{29}$. Usually, the DEJ separation increases with the degree of demineralization. In a study of SS-OCT, the gaps along DEJ were first found after 1 week of demineralization and slightly increased after the extension of the experimental period, which implied the integrity of DEJ was considered to be vulnerable to the carious demineralization ${ }^{20)}$.

This study also showed a negative aspect that resin infiltration had risks of cervical enamel loss and dentin over removal because of the strong acid $(15 \% \mathrm{HCl})$ pretreatment. Although resin infiltration (pretreated by $120 \mathrm{~s} \mathrm{HCl}$ ) had less lesion depth increase than the control group and deeper resin penetration into the lesion body and DEJ separation than SE Bond, more specimens in caries-infiltrant groups (pretreated by $\mathrm{HCl}$ for $120 \mathrm{~s}$ and for $10 \mathrm{~s}$ ) had cervical enamel loss than the specimens in SE Bond group. The stronger acid with a longer time application caused more damage to the normal dental hard tissues. In case of soft tissues, although short-term contact of this strong acid with mucosa has been shown to be harmless ${ }^{30}$, it may cause severe burns on contact with soft tissues even with a rubber dam in clinical practices. Also, a strong acid can cause sensitivity on exposed dentin surface after a gingival recession.

Propidium iodide (PI, LIVE/DEAD BacLight Bacterial Viability Kit), the red-fluorescent nucleic acid stain is usually used to detect dead bacteria and also dead animal cells. In the present study, PI was used as a fluorescent dye mixed with Icon-Infiltrant or with SE Bond. We successfully detected them by a FM to get clear images inside the demineralized dentin body (as PI has an affinity to bind with resin and has been used in ion-exchange biotechnology at a molecular level). There are some discrepancies in resin infiltration that caused incomplete and inhomogeneous filling into lesions. The caries model formed on human teeth using the major cariogenic bacteria by simulating an oral environment in this study expected to be very similar to the natural caries. Therefore, incomplete surface layer erosion (even for those etched with $\mathrm{HCl}$ ) in natural lesions and organic materials, such as proteins and carbohydrates, which might contaminate the pores of natural caries, could be responsible for those discrepancies ${ }^{31}$. Moreover, inorganic components of dentin (e.g. collagens) might have resisted proper penetration of the resin-infiltrant and interfered its solidification as it happens in case of non-cavited enamel chemically combining with enamelprisms. Other factors, including residual biofilms, bacteria, culture media, other solutions, residual chemical compounds, reduced wettability and entrapped air might hamper resin penetration as well.

Four specimens in each group were stained instead of all specimens because the main purpose was to evaluate the lesion depth using SS-OCT considering that mixing with the stain may cause some disturbances to the infiltrant, that did not occur actually though. Another technical difficulty of this staining was that the FM images needed to be taken just after the material application and before the second biofilm attack. Moreover, the substructure for the application of resin infiltration was caries-infected dentin rather than noncavitated enamel caries. The current version of Iconinfiltant is actually indicated for early enamel caries treatment only, not for root caries treatment; rather contra-indicated under the same application protocol of pretreatment with $120 \mathrm{~s} 15 \% \mathrm{HCl}$. As obvious, risks of damaging delicate structures at the cervical parts of vital or even non-vital teeth have been considered. To minimize those risks, the application of strong acids for the shortest possible time (10 s) was also tested in this study. Unfortunately, results were not expectedly effective both in terms of the resin penetration and the prevention of demineralization progression. However, this is the first study till now to evaluate the effect of resin infiltration on root caries, which may provide some possibilities and information to explore a wider application of the material.

Consequently, modified protocols and studies on 
the pretreatments for cervical hard and soft tissues protection and for root caries against the further demineralization seem to be necessary for the application of resin infiltration in future.

\section{CONCLUSION}

Within the limitations of this in vitro study, it can be concluded that resin infiltration pretreated by $120 \mathrm{~s} \mathrm{HCl}$ has a better penetration ability than that pretreated by $\mathrm{H}_{3} \mathrm{PO}_{4}$ and SE Bond. Also it has a preventive effect on root caries as similar as SE Bond. However, the strong acid $(15 \% \mathrm{HCl})$ pretreatment has risks of cervical enamel loss and over removal of dentin structure as well.

\section{ACKNOWLEDGMENTS}

This research was supported by the Research Grant for longevity sciences (21A-8) from Japanese Ministry of Health, Labor and Welfare and by Grant-in-Aid for Scientific Research (16K11544) from the Japan Society for the Promotion of Science (JSPS).

\section{REFERENCES}

1) Arthur RA, Martins VB, de Oliveira CL, Leitune VC, Collares FM, Magalhães AC, Maltz M. Effect of over-the-counter fluoridated products regimens on root caries inhibition. Arch Oral Biol 2015; 60: 1588-1594.

2) Murray CG. Advanced restorative dentistry -a problem for the elderly? An ethical dilemma. Aust Dent J 2015; 60(suppl 1): 106-113.

3) Burgess JO, Gallo JR. Treating root-surface caries. Dent Clin North Am 2002; 46: 385-404.

4) Banting DW, Papas A, Clark DC, Proskin HM, Schultz M, Perry R. The effectiveness of $10 \%$ chlorhexidine varnish treatment on dental caries incidence in adults with dry mouth. Gerodontology 2000; 17: 67-76.

5) Närhi TO, Meurman JH, Ainamo A. Xerostomia and hyposalivation: causes, consequences and treatment in the elderly. Drugs Aging 1999; 15: 103-116.

6) Rolland SL, Walls AW, McCabe JF, German MJ. Use of micro-Raman spectroscopy to investigate hybrid layer quality in demineralized root dentine. J Biomed Mater Res B Appl Biomater 2010; 95: 62-68.

7) Ma S, Imazato S, Chen JH, Mayanagi G, Takahashi N, Ishimoto T, Nakano T. Effects of a coating resin containing S-PRG filler to prevent demineralization of root surfaces. Dent Mater J 2012; 31: 909-915.

8) Gando I, Ariyoshi M, Ikeda M, Sadr A, Nikaido T, Tagami J. Resistance of dentin coating materials against abrasion by toothbrush. Dent Mater J 2013; 32: 68-74.

9) Ekstrand KR, Bakhshandeh A, Martignon S. Treatment of proximal superficial caries lesions on primary molar teeth with resin infiltration and fluoride varnish versus fluoride varnish only: efficacy after 1 year. Caries Res 2010; 44: 4146.

10) Martignon S, Ekstrand KR, Gomez J, Lara JS, Cortes A. Infiltrating/sealing proximal caries lesions: a 3-year randomized clinical trial. J Dent Res 2012; 91: 288-292.

11) Meyer-Lueckel H, Bitter K, Paris S. Randomized controlled clinical trial on proximal caries infiltration: three-year followup. Caries Res 2012; 46: 544-548.

12) Paris S, Schwendicke F, Seddig S, Müller WD, Dörfer C, Meyer-Lueckel H. Micro-hardness and mineral loss of enamel lesions after infiltration with various resins: influence of infiltrant composition and application frequency in vitro. $\mathrm{J}$ Dent 2013; 41: 543-548.

13) Paris S, Meyer-Lueckel H, Cölfen H, Kielbassa AM. Resin infiltration of artificial enamel caries lesions with experimental light curing resins. Dent Mater J 2007; 26: 582588.

14) Meyer-Lueckel H, Paris S. Progression of artificial enamel caries lesions after infiltration with experimental light curing resins. Caries Res 2008; 42: 117-124.

15) Meyer-Lueckel H, Paris S. Improved resin infiltration of natural caries lesions. J Dent Res 2008; 87: 1112-1116.

16) Meyer-Lueckel H, Paris S, Mueller J, Cölfen H, Kielbassa AM. Influence of the application time on the penetration of different dental adhesives and a fissure sealant into artificial subsurface lesions in bovine enamel. Dent Mater 2006; 22: 22-28.

17) Paris S, Meyer-Lueckel H. Inhibition of caries progression by resin infiltration in situ. Caries Res 2010; 44: 47-54.

18) Huang D, Swanson EA, Lin CP, Schuman JS, Stinson WG, Chang W, Hee MR, Flotte T, Gregory K, Puliafito CA, Fujimoto JG. Optical coherence tomography. Science 1991; 254: 1178-1181.

19) Mandurah MM, Sadr A, Shimada Y, Kitasako Y, Nakashima S, Bakhsh TA, Tagami J, Sumi Y. Monitoring remineralization of enamel subsurface lesions by optical coherence tomography. J Biomed Opt 2013; 18: 046006.

20) Tezuka H, Shimada Y, Matin K, Ikeda M, Sadr A, Sumi Y, Tagami J. Assessment of cervical demineralization induced by Streptococcus mutans using swept-source optical coherence tomography. J Med Imaging (Bellingham) 2016; 3: 014504.

21) Min JH, Inaba D, Kwon HK, Chung JH, Kim BI. Evaluation of penetration effect of resin infiltrant using optical coherence tomography. J Dent 2015; 43: 720-725.

22) Hayati F, Okada A, Kitasako Y, Tagami J, Matin K. An artificial biofilm induced secondary caries model for in vitro studies. Aust Dent J 2011; 56: 40-47.

23) Daneshmehr L, Matin K, Nikaido T, Tagami J. Effects of root dentin surface coating with all-in-one adhesive materials on biofilm adherence. J Dent 2008; 36: 33-41.

24) Natsume Y, Nakashima S, Sadr A, Shimada Y, Tagami J, Sumi Y. Estimation of lesion progress in artificial root caries by swept source optical coherence tomography in comparison to transverse microradiography. J Biomed Opt 2011; 16: 071408.

25) Ammari MM, Soviero VM, da Silva Fidalgo TK, Lenzi M, Ferreira DM, Mattos CT, de Souza IP, Maia LC. Is noncavitated proximal lesion sealing an effective method for caries control in primary and permanent teeth? A systematic review and meta-analysis. J Dent 2014; 42: 1217-1227.

26) Sugawara T, Nakashima S, Shimizu A, Tagami J, Momoi Y. Evaluation of a new hardness tester (Cariotester): Comparison with transverse microradiography for assessing the inhibitory effect of fluoride application on bovine root dentin demineralization. Dent Mater J 2015; 34: 371-378.

27) Paris S, Lausch J, Selje T, Dörfer CE, Meyer-Lueckel H. Comparison of sealant and infiltrant penetration into pit and fissure caries lesions in vitro. J Dent 2014; 42: 432-438.

28) Meyer-Lueckel H, Paris S, Kielbassa AM. Surface layer erosion of natural caries lesions with phosphoric and hydrochloric acid gels in preparation for resin infiltration. Caries Res 2007; 41: 223-230.

29) Imai K, Shimada Y, Sadr A, Sumi Y, Tagami J. Noninvasive cross-sectional visualization of enamel cracks by optical coherence tomography in vitro. J Endod 2012; 38: 1269-1274.

30) Croll TP, Killian CM, Miller AS. Effect of enamel microabrasion compound on human gingiva: report of a case. Quintessence Int 1990; 21: 959-963.

31) Paris S, Meyer-Lueckel H, Kielbassa AM. Resin infiltration of natural caries lesions. J Dent Res 2007; 86: 662-666. 